\title{
AN EFFICIENT SIGNAL REDISTRIBUTION ALGORITHM FOR MCM
}

\author{
Ming-Fu Shiao, Chieh Changfan, Sao-Jie Chen, *Chia-Chun Tsai \\ Dept. of Electrical Engineering \\ National Taiwan University \\ Taipei, Taiwan, R.O.C. \\ *Dept. of Electronic Engineering \\ National Taipei Institute of Tech. \\ Taipei, Taiwan, R.O.C.
}

\begin{abstract}
In a Multichip Module (MCM), the I/O pins of dies are redistributed to signal layers before routing. In this paper, a new model based on congestion control was proposed to solve this problem. A 2-layer global and detailed router was employeed to finish the routing. And experimental results show that this model can produce very routable pin distribution in each layer for Multichip Module.
\end{abstract}

\section{INTRODUCTION}

In a Multichip Module ( $M C M$ ), several bare dies are mounted on the top layer of a laminated multilayer ceramic or a thin substrate. The substrate provides metal wiring for the signal as well as for the power and ground connections. The elimination of individual chip package and direct connections of bare dies can significantly reduce the inter-chip delay, weight, size, and hence promotes the entire system performance.

This paper deals with the signal redistribution of MCM-C (multilayer ceramic), but it can also be applied to MCM-D(thin film module) and PCBs. In Section 2, the signal redistribution problem is formulated. In Section 3, a congestion control model is derived to evaluate the congestion of each layer. In Section 4, a redistribution algorithm based on the congestion model is used to solve this problem. Experimental results and conclusion are described in Section 5 .

\section{PROBLEM FORMULATION}

In Multichip Modules, the I/O pins of mounted dies have to be redistributed to the signal layers to facilitate routing. A good redistribution of $\mathrm{I} / \mathrm{O}$ pins can significantly reduce the signal layers needed for routing as well as increase the wirability of each layer. Typically, the layer structure of a multilayer MCM is arranged as shown in Figure 1. Neighboring $\mathrm{X}$ - and $\mathrm{Y}$-signal layers construct a local routing region. The redistribution problem is formulated as follows:

tThis Paper is supported in part by the National Science Council R.O.C. under Grants NSC 82-0404-E002-166, and NSC 82-0404-E027-020
Given a set of nets $N_{1}, N_{2}, . . N_{n}$, and a set of pins $P_{i}$ which belongs to $N_{1}, N_{2}, . . N_{n}$, a substrate which consists of $2 m$ signal layers is used to connect all these nets. We want to find a transformation function

$$
\begin{gathered}
T\left(P_{i}\right)=j \\
\text { where } 1 \leq j \leq m
\end{gathered}
$$

such that

(1) all nets can be connected in the $2 m$ layers,

(2) the use of $m$ is minimized.

There are many possible ways to solve this problem. One popular way is to pre-generate two-dimensional wiring routes for all nets, and then, assign all the wires to layers such that intersecting wires of different nets are assigned to distinct layers [1] [2]. The other possible way is to partition all the nets into groups based on some criteria, and then route each group of nets in a local routing region independently. This method does not generate the topologies of nets in advance, and hence has more flexibility in wiring topologies.

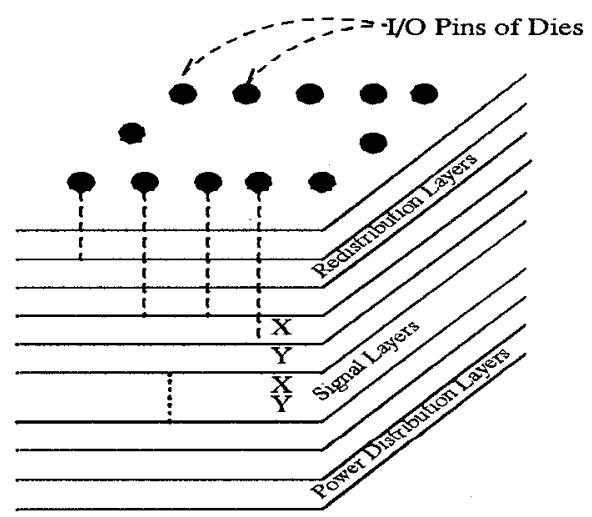

Figure 1: Layer structure of a multilayer Multichip Module, where neighboring $\mathrm{X}$ - and $\mathrm{Y}$-signal layers construct a local routing region.

\subsection{The MCM Multilayer Routing Model}

Multilayer routing problem has been studied extensively since the age of $\mathrm{PCB}$. The routing model of $\mathrm{M}$ - 
$\mathrm{CM}$ is analogous to that of PCB except the following variations: First, most $\mathrm{PCB}$ technologies place chips using drilled through hole, i.e., all signal layers can access the $\mathrm{I} / \mathrm{O}$ pins of chips. While in MCM, only the top layer bears the mounted dies. The $1 / O$ pins of dies have to be redistributed to the signal layers before routing. Second, recent MCM technologies support different types of vias. While PCBs only supports vias of Plated-Through-Hole (PTH).

Generally, there are three different types of vias used in MCM [3]. A pass through via connects signal from the top layer to the bottom one. This kind of vias usually connect I/O pins of dies to the $I / O$ pads of the entire module. A segmented via defined as a via pass through a subset of wiring planes, serves the role of distributing signals. A programmable via interconnects wires on two adjacent wiring planes, and can be placed at any grid location. That is, vias of different nets can be placed on the same grid location of different layers. Figure 2 shows these three types of vias.

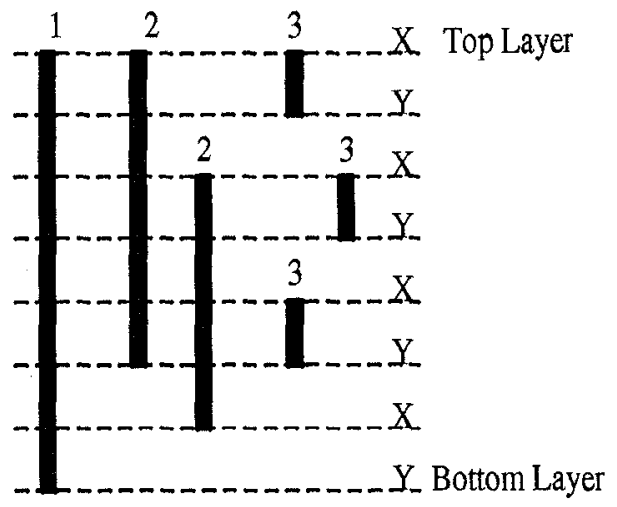

Figure 2: Three types of vias: Type 1, pass through via; Type 2, segmented via; Type 3 , programmable via

\section{CONGESTION MODEL}

\subsection{Net Split}

If a net consists of too many pins, it could cause potential congestion in many layers. Since this kind of nets are usually split into subnets and assigned to distinct layers, the pins of a subnet must be localized in a small area in order to minimize the wiring length and potential congestion. A practical split procedure based on constrained clustering is proposed as follows.

First, in order to localize the pins of a subnet. We define a threshold value Max_Mass_Pin_Ratio (MM$\mathrm{PR}$ ), such that the mass_pin_ratio of all subnets must not exceed this value. Second, a pair of pins which has the farest distance is selected as the seeds. These seed pins start to merge the pins nearest to it one by one until all the pins are merged. Another cycle is started while the mass_pin_ratio of some split subnets

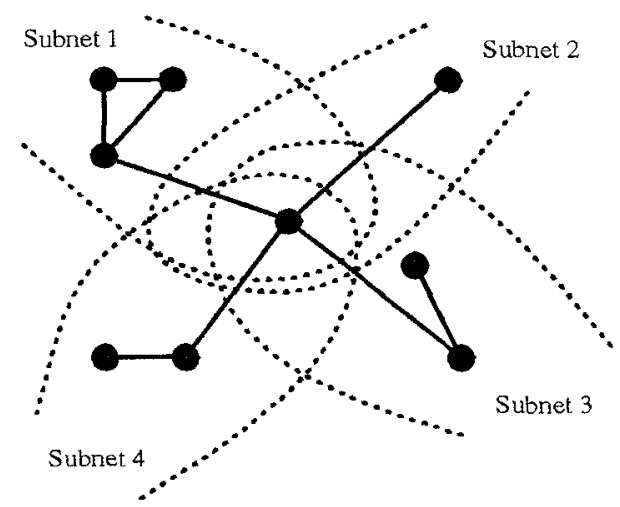

Figure 3: A large net is split into 4 subnets

still exceed the MMPR. The cycle will stop until no subnet has a mass_pin_ratio greater than the MMPR.

After the net split, all subnets are regarded as independent nets. Figure 3 shows an example of a split net.

\subsection{Force Model}

After the net split, all subnets are limited in a local region. In the next phase, subnets are partitioned into several groups such that each group can be routed in a local routing region. To achieve a routable group, each subnet of the same group must have the minimum influence on each other. To capture this scenario, a force model is proposed as the following:

A net $n$ which contains $P$ pins is regarded as a mass point with mass and center of mass point where the center of this mass point is defined as:

$$
\left(\bar{X}_{n}, \bar{Y}_{n}\right)=\left(\frac{\sum_{p i \in n} X_{p i}}{P}, \frac{\sum_{p i \in n} Y_{p i}}{P}\right)
$$

And the mass is defined as:

$$
M_{n}=\sum_{p i \in n}\left(\left(X_{p i}-\bar{X}_{n}\right)^{2}+\left(Y_{p i}-\bar{Y}_{n}\right)^{2}\right)
$$

where $X_{p i}, Y_{p i}$ are the $\mathrm{X}$ - and $\mathrm{Y}$-coordinate of the $i$ 'th pin of net $n$ respectively.

The induced force of two nets $N_{i}, N_{j}$ is defined as

$$
\operatorname{Force}\left(N_{i}, N_{j}\right)=\frac{M_{i} * M_{j}}{\operatorname{Distance}\left(N_{i}, N_{j}\right)^{2}}
$$

where $M_{i}, M_{j}$ are the masses of net $i$, net $j$ respectively.

$$
\operatorname{Distance}\left(N_{i}, N_{j}\right)=\sqrt{\left(\bar{X}_{i}-\bar{X}_{j}\right)^{2}+\left(\bar{Y}_{i}-\bar{Y}_{j}\right)^{2}}
$$

Before the assignment, the induced force of a subnet to a routing region is calculated in advance. If a net $N$ 


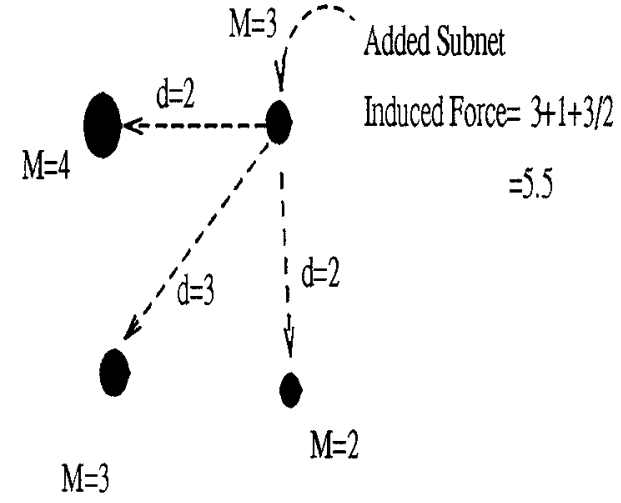

Figure 4: The force induced by an added subnets

is assigned to a routing region $R R$, the induced force would be:

$$
\operatorname{Force}(N, R R)=\sum_{j \in R R} \operatorname{Force}\left(N, N_{j}\right)
$$

Obviously, if the induced force of a net is strong, this net is potentially congested in this routing region. Hence the pending subnet prefers being assigned to a layer which is the most sparse. Figure 4 shows an example of induced force by a newly added subnet.

\subsection{Capacity Estimation}

A capacity estimation function is used to evaluate the available tracks while a subnet is assigned to a routing region. And this estimation function provides a penalty in the cost function. This estimation function is initially defined as the following:

$$
\begin{aligned}
& C_{x, 0}=E_{x} *(A V X) \\
& C_{y, 0}=E_{y} *(A V Y)
\end{aligned}
$$

where $A V X, A V Y$ is the available $X$ tracks and $Y$ tracks of this routing region respectively. $E_{x}, E_{y}$ which range from 0 to 1 are the user defined $X$-track and $Y$-track densities for each routing region. If this value is low, that is, the cost in capacity penalty is high, such that all subnets are not likely to reside on this layer. Normally, the top layers have higher value of $E_{x}, E_{y}$ than the bottom layers. If a subnet $n$ is assigned to a routing region $\mathrm{RR}$, the penalty will be:

$C P($ Net $n, R R)=$

$\left[\frac{1}{C_{x, n}-\sum_{p i \in n}\left(X_{p i}-\bar{X}_{n}\right)}+\frac{1}{C_{y, n}-\sum_{p i \in n}\left(Y_{p i}-\bar{Y}_{n}\right)}\right]^{W 1}$

After assignment, the capacity will become:

$$
\begin{aligned}
& C_{x, n+1}=C_{x, n}-\sum_{p i \in n}\left(X_{p i}-\bar{X}_{n}\right) \\
& C_{y, n+1}=C_{y, n}-\sum_{p i \in n}\left(Y_{p i}-\bar{Y}_{n}\right)
\end{aligned}
$$

\section{REDISTRIBUTION ALGORITHM}

The cost function of a pending subnet corresponding to a routing region $R R$ is defined as the sum of the induced force and capacity penalty. The force cost balances the congestion of layers, and the capacity penalty balances the track usage of each layer. Therefore, for a given subnet and a routing region, the induced cost is:

$\operatorname{Cost}\left(N_{i}, R R\right)=C P\left(N_{i}, R R\right)+W 2 * \operatorname{Force}\left(N_{i}, R R\right)$

where $W 2$ is the weight of the induced force. The redistribution procedure is listed below.

\section{Procedure Signal Redistribution \\ Net_Split(all nets) \\ For $(i=1$ to no. of unassigned nets) \\ For $(j=1$ to no. of routing regions)}

Calculate the cost of net $i$ in routing region $j$

End For

Select a routing region with the minimum cost

Assign net $i$ to the selected region

End For

End Signal_Redistribution

\section{RESULTS AND CONCLUSION}

\subsection{Experimental Results}

Three circuits which contain pins from 400 to 3200 are randomly generated and used to test the algorith$\mathrm{m}$. The results of signal redistribution algorithm are routed by an integrated routing tool [4] [5], which combines the 2-layer global routing and detailed routing. Table 1 lists the results, and Figure 5 shows the final routing of a sample circuit, which has 900 pins in the top layer and where six layers are used to complete the final routing.

Table 1: Experimental Results

\begin{tabular}{|c|c|c|c|}
\hline Circuit & MCM 400 & MCM 900 & MCM 3200 \\
\hline Net & 80 & 200 & 800 \\
\hline Pin & 400 & 900 & 3200 \\
\hline Grid & $90 \times 90$ & $170 \times 170$ & $320 \times 180$ \\
\hline Layers & 6 & 6 & 12 \\
\hline Vias & 2027 & 4882 & 19108 \\
\hline Wire Length & 12696 & 55806 & 160078 \\
\hline
\end{tabular}

\subsection{Conclusion}

A new concept of signal redistribution strategy is introduced in this paper. And this method has the following advantages. (1) By using our net split and redistribution algorithm, a $2 m$-layer routing problem is reduced to an $m$ 2-layer routing problem, which will significantly reduce the complexity of MCM routing problem. (2) The high routability pin distribution in each layer is derived by the congestion control which reduces the total number of signal layers needed to complete the final routing. (3) After the signal redistribution, the final routing of local routing regions can be done independently. This is a good characteristic for parallel processing. 


\section{References}

[1] J.M.Ho, M.Sarrafzadeh, G.Vijayan, C.K.Wong, "Layer Assignment for Multichip Modules," IEEE Trans. CAD, CAD-9(12), pp.1272-1277, Dec. 1990.

[2] M.Sriram and S.M.Kang, "Detailed Layer A.ssignment for MCM Routing," in Proceedings of the International Conference on Computer Aided Design, pp.386-389, Nov. 1992.

[3] R.R.Tummala, "Ceramic Packaging", in Microelectronics Packaging Handbook, edited by R.R.Tummala and E.J.Rymaszewski, van Nostrand Reinhold, New York, 1989, pp.455-522.

[4] T.M.Parng and R.S.Tay, "A new approach to seaof-gate global routing," in Proceedings of the International Conference on Computer-Aided Design, pp.52-55, 1989.

[5] W.C.Kao, A tool bridging the gap between global and detailed routing, M.S. thesis, Dept. of Electrical Engineering, National Taiwan University, R.O.C., 1992.

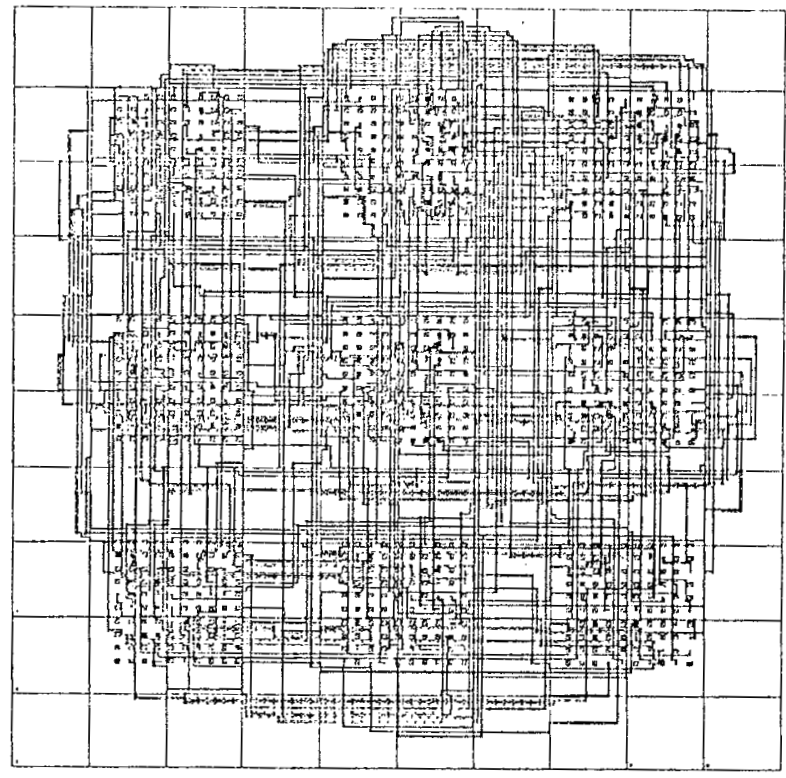

(a)

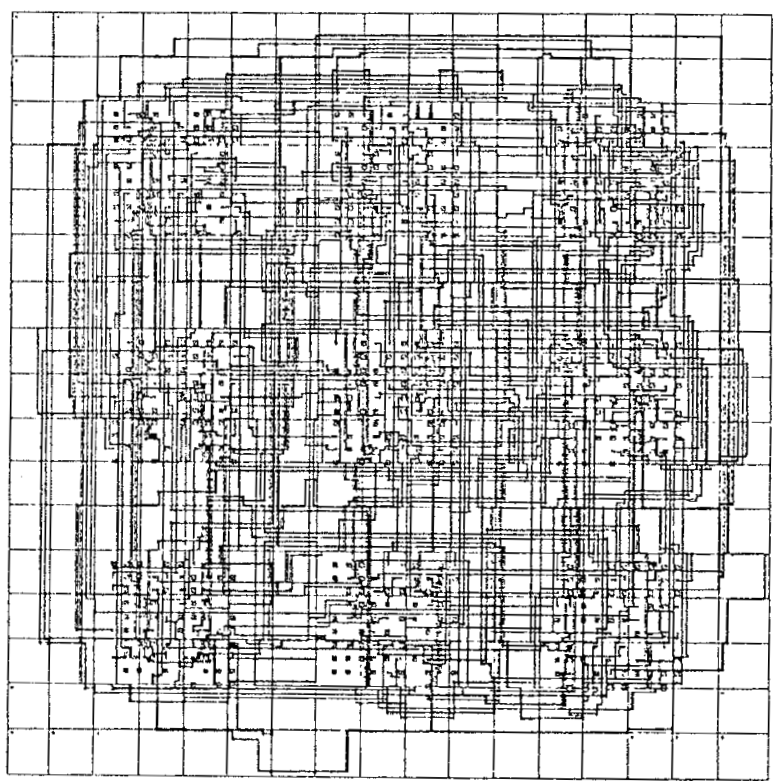

(b)

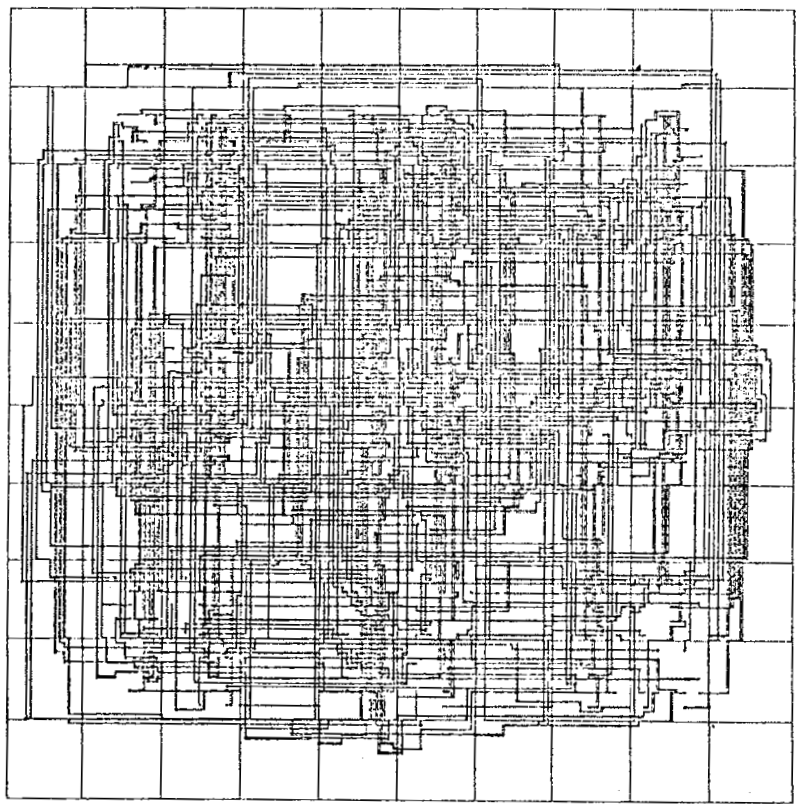

(c)

Figure 5: The final routing of a sample circuit which has 900 pins and six signal layers. (a) the top routing region (b) middle. (c) the bottom routing region. 